\title{
Fluorescence excitation analysis by two-photon confocal laser scanning microscopy: a new method to identify fluorescent nanoparticles on histological tissue sections
}

\author{
This article was published in the following Dove Press journal: \\ International Journal of Nanomedicine \\ 19 October 2012 \\ Number of times this article has been viewed
}

\author{
Edmond Kahn' \\ Nicolas Tissot ${ }^{3}$ \\ Perrine Frere ${ }^{3}$ \\ Aurélien Dauphin ${ }^{3}$ \\ Mohamed Boumhras ${ }^{2,4}$ \\ Claude-Marie Bachelet ${ }^{3}$ \\ Frédérique Frouin' \\ Gérard Lizard ${ }^{2}$ \\ 'Institut National de la Santé et de \\ la Recherche Médicale (INSERM) \\ U678/UMR-S UPMC, CHU Pitié- \\ Salpêtrière, Paris, France; ${ }^{2}$ Equipe \\ Biochimie du Peroxysome, \\ Inflammation et Métabolisme Lipidique \\ EA7270, Faculté des Sciences Gabriel, \\ Université de Bourgogne-INSERM \\ Dijon, France; ${ }^{3}$ Plateforme d'Imagerie \\ cellulaire, UPMC, Paris, France; \\ ${ }^{4}$ Laboratory of Biochemistry and \\ Neuroscience, Applied Toxicology \\ Group, Faculty of Science and \\ Technology, Settat, Morocco
}

\begin{abstract}
In the present study, we make use of the ability of two-photon confocal laser scanning microscopes (CLSMs) equipped with tunable lasers to produce spectral excitation image sequences. Furthermore, unmixing, which is usually performed on emission image sequences, is performed on these excitation image sequences. We use factor analysis of medical image sequences (FAMIS), which produces factor images, to unmix spectral image sequences of stained structures in tissue sections to provide images of characterized stained cellular structures. This new approach is applied to histological tissue sections of mouse aorta containing labeled iron nanoparticles stained with Texas Red and counterstained with SYTO13, to obtain visual information about the accumulation of these nanoparticles in the arterial wall. The possible presence of Texas Red is determined using a two-photon CLSM associated with FAMIS via the excitation spectra. Texas Red and SYTO13 are thus differentiated, and corresponding factor images specify their possible presence and cellular localization. In conclusion, the designed protocol shows that sequences of images obtained by excitation in a two-photon CLSM enables characterization of Texas Red-stained nanoparticles and other markers. This methodology offers an alternative and complementary solution to the conventional use of emission spectra unmixing to localize fluorescent nanoparticles in tissue samples.
\end{abstract}

Keywords: FAMIS, spectral excitation sequences, Texas Red, tunable excitation, unmixing

\section{Introduction}

In the present study, we make use of the ability of two-photon confocal laser scanning microscopes (CLSMs) equipped with tunable lasers to produce spectral excitation image sequences. The added benefit over most previous spectral confocal studies, which have been carried out by analyzing emission spectra only, is that the extracted spectrum that is successful in unmixing a particular stained structure is provided through the examination of the shape of its excitation properties. ${ }^{1}$ In our study, factor analysis of medical image sequences (FAMIS), ${ }^{2,3}$ which combines correspondence analysis and oblique analysis and provides factor images of stained structures, handles mixtures of components determined by their physical behaviors in the sequence. ${ }^{4,5}$ FAMIS performs unmixing through spectral image processing of fluorescent stained structures on tissue sections and provides characterized stained cellular structures. Unmixing can be performed on emission spectral image sequences via conventional techniques, which are available in most confocal microscopes. ${ }^{6}$ Spectral analysis is by itself a broad field. A few of the more basic algorithms in spectral
Correspondence: Edmond Kahn INSERM U678/UMR-S UPMC, CHU Pitié-Salpêtrière,

75634 Paris Cedex 13, France

$\mathrm{Tel}+33$ | 53828443

Fax +33 । 53828448

Email kahn@imed.jussieu.fr 
imaging are described by Garini, Young, and McNamara. ${ }^{7}$ Unmixing can also be performed on excitation spectral image sequences, as tunable lasers enable automated image acquisition at multiple excitation wavelengths, ${ }^{8}$ providing additional information about fluorescent structures inside tissue sections. To illustrate this, we applied unmixing protocols including FAMIS to histological tissue sections of mouse aorta containing Texas Red-labeled iron (Fe) nanoparticles counterstained with SYTO13, ${ }^{9}$ to get visual information on the accumulation of these nanoparticles in the arterial wall. Our aim was to establish the ability of fluorescent MRI contrast agents to act as in vivo staining tools for cellular sites. Texas Red-labeled Fe nanoparticles have been developed to mimic regular ultrasmallparticle iron oxide contrast agents.

Preliminary experiments were performed on specimens containing calibrated beads and Texas Red fluorescent nanoparticles ${ }^{10}$ to enhance the value of this new method based on tunable excitation.

In our experimental conditions, sequences of images obtained through a two-photon CLSM are processed by FAMIS, which unmixes fluorochromes on the basis of their physical properties ${ }^{11}$ and thus enables the isolation and visualization of fluorochromes by their spectral patterns. ${ }^{12,13}$ The fluorochromes in question are Texas Red and SYTO13. We also consider fluorescein isothiocyanate (FITC), because the selected calibrated beads contain a mixture of Texas Red and FITC. The excitation sequences of images are processed according to a method based on FAMIS that is available via Pixies (see http://www.apteryx.fr/). Sequences of images are obtained according to a protocol that requires the memorization of the scanning along the excitation spectrum (705-965 nm). The aim of the study is to differentiate the excitation spectra of Texas Red-stained and SYTO13-stained structures to determine precisely their localization in arterial wall histological tissue sections (thoracic aorta of mice) by using computed excitation curves (factors) and corresponding factor images.

\section{Materials and methods}

\section{Specimens}

As prior controls, we selected (1) FTR beads (Flow Cytometry Standards Corporation, Research Triangle Park, NC), combining Texas Red and FITC, as a physical model; and (2) MACS Fe nanoparticles, which are goat anti-rabbit immunoglobulin G (IgG) microbeads (130-048-602; Miltenyi Biotec, Bergisch Gladbach, Germany) incubated with Texas Red-conjugated rabbit IgG (011-0902; Rockland Immunochemicals, Gilbertsville, PA) in order to obtain fluorescent nanoparticles. ${ }^{10}$
To provide a biological specimen, a product combining Fe nanoparticles and Texas Red (MRC 98145; Institute für Diagnostik Forschung, Berlin, Germany) was injected into the vein of the tail of Balb/c female mice (10-13 weeks). ${ }^{9}$ The concentration of the injected product was similar to that used for clinical purposes in humans $(0.05 \mathrm{mM} / \mathrm{kg})$. A noninjected mouse was used as a negative control. Mice were killed 4 minutes after injection by cervical dislocation. Fragments of fresh thoracic aorta of the mice were prepared for CLSM processing, and the cell nuclei of tissue samples were counterstained with SYTO13 as described previously. ${ }^{9}$

\section{Observation by two-photon CLSM fluorescence analysis}

Specimens were observed using two-photon CLSM (Zeiss LSM 710 NLO; Carl Zeiss, Oberkochen, Germany), equipped with ScanModule (Carl Zeiss), photomultipliers, and nondescanned detectors. The emissions and excitations of specimens containing MACS Texas Red Fe particles, FTR beads, and injected MRC Texas Red Fe particles and counterstained SYTO13 were analyzed by means of a two-photon CLSM in sequences of images obtained by the available spectral analysis settings, which include an automatic spectral mode. Images were obtained for each case in $512 \times 512$ matrices at 20-times magnification (WPlanApochromat $20 \times / 1.0$ ) of the two-photon CLSM (selected pixel sizes from 0.14 to $0.83 \mu \mathrm{m}$ ). Excitation was carried out with a tunable Titanium Sapphire laser (Chameleon Ultra $2+$ precompensation; Coherent). The acquisition time to scan each image was about 1 second, and the time required to change an excitation wavelength in the automatic spectral mode was about 2 seconds. An excitation at $805 \mathrm{~nm}$ was performed to collect the emission produced in the $10 \mathrm{~nm}$ spectral mode from blue to red (398 to $718 \mathrm{~nm}$ ). Excitation spectral sequences were acquired in $512 \times 512$ matrices at $20 \times$ magnification in sequences of 27 images, in the $705-965 \mathrm{~nm}$ excitation range, in $10 \mathrm{~nm}$ steps, without filters.

In the case of spectral observations in which emissions were collected through band-pass filters and processed before interpretation, the excitation at $805 \mathrm{~nm}$ was performed on the basis of emission signal intensity, noting that excitation is recommended at $780 \mathrm{~nm}$ for Texas Red, ${ }^{14}$ between $740 \mathrm{~nm}$ and $820 \mathrm{~nm}$ for FITC, and at $810 \mathrm{~nm}$ for SYTO13. Red emissions were interpreted as Texas Red (peak reported between 596 and $620 \mathrm{~nm}),{ }^{14}$ and green emissions were interpreted as autofluorescence and FITC (peak reported at $519 \mathrm{~nm}$ ). ${ }^{14}$ 
In the case of spectral observations in which excitations were collected in the spectral mode from 705 to $965 \mathrm{~nm}$, excitation peaks corresponding to Texas Red and to FITC and autofluorescence were obtained by means of FAMIS (see Image analysis), bearing in mind the recommended excitation levels. ${ }^{14}$ Images were superimposed in their true colors to provide a supplementary tool for interpretation.

\section{Image analysis}

Following general factor analysis techniques, FAMIS was developed to process biomedical image sequences. ${ }^{2,3}$ FAMIS decomposes image sequences into a smaller number of images, called factor images, and curves, called factor curves. ${ }^{15,16}$ Factor images correspond to spatial distribution components, while factor curves estimate individual behaviors in the sequence of images. The basic idea of FAMIS is to process the curves that represent the evolution of fluorescence intensity of each pixel in the spectral emission image, or excitation (Figure 1). The analysis assumes that each pixel is a mixture of fluorescence patterns, and the aim is to unmix those patterns. Factors are estimated from the image sequence in a two-step procedure. In the first step, called correspondence analysis, the image is reconstituted using singular vectors associated with the largest singular values. The second step, called oblique analysis, aims to estimate factor curves representing the fundamental curves. An algorithmic description of FAMIS is provided in a previous publication by Kahn et al. ${ }^{16}$

Using FAMIS, pixel images were recombined in $4 \times 4$ clusters. All such clusters were investigated. Correspondence analysis ${ }^{17,18}$ was first performed on the intensity evolution of each cluster; then oblique analysis with positivity constraints was performed on the results of the correspondence analysis $^{2}$ to obtain positive factor curves and images. Factor

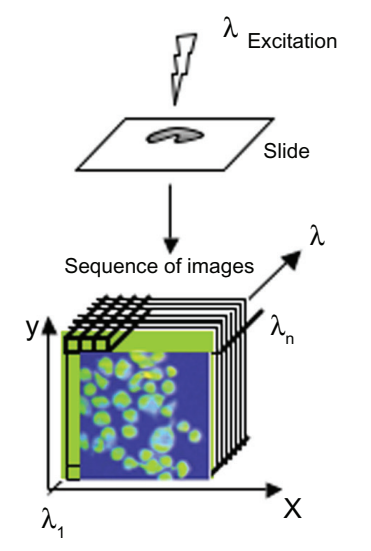

Estimation of factor images

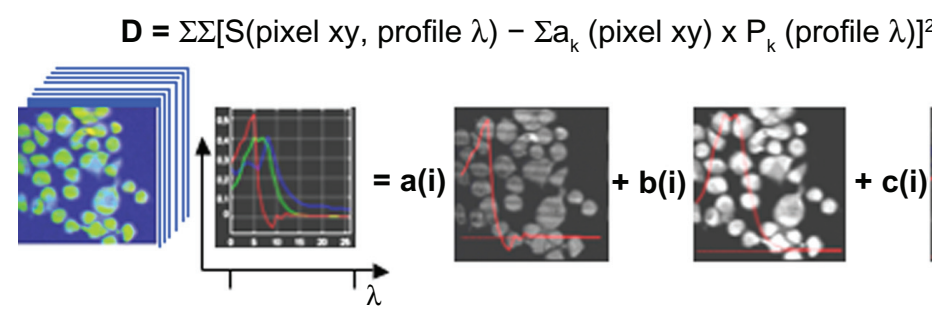

$\mathbf{D}=\Sigma \Sigma\left[\mathrm{S}(\text { pixel } \mathrm{xy}, \text { profile } \lambda)-\Sigma \mathrm{a}_{\mathrm{k}}(\text { pixel } \mathrm{xy}) \times \mathrm{P}_{\mathrm{k}}(\text { profile } \lambda)\right]^{2}$

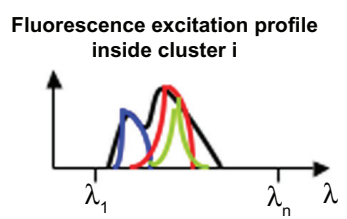

Fluorescence excitation profile

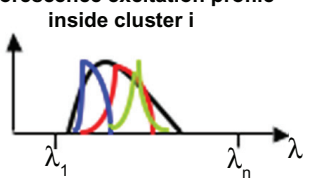

Orthogonal analysis

Singular value decomposition of matrix $X(N, P)$

Cluster i j $\quad$ j $\quad p$

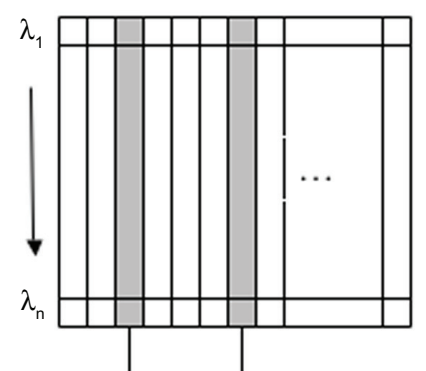

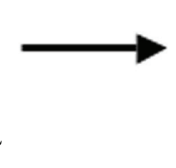

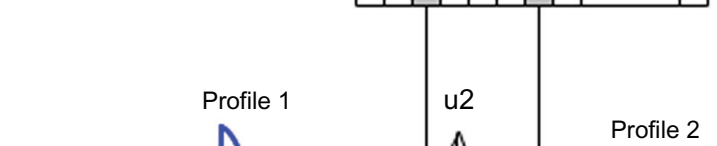

Profile 2

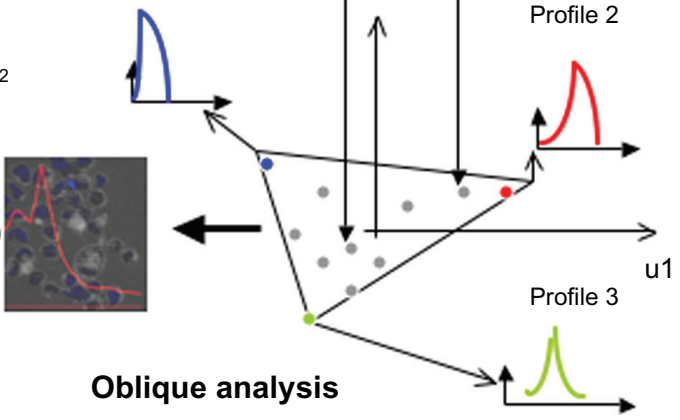

Figure I Basic presentation of FAMIS, which was developed to process biomedical image sequences. ${ }^{2,3}$ A similar description of FAMIS applied to spectral emission was provided in Kahn et al ${ }^{10}$

Notes: In the present case, FAMIS assumes that each pixel is a mixture of different fluorescence patterns. ${ }^{5}$ Factor curves correspond to spectral emissions ${ }^{13,15}$ of the different fluorochromes on the slide. Here, factor curves correspond to spectral excitations of the fluorochromes. For each factor curve, the set of positive weights computed for each curve yields one image, called a factor image, which provides images of fluorescent structures. Factors are estimated in a two-step procedure from the image sequence. In the first step, called correspondence analysis, the image is reconstituted using singular vectors associated with the largest singular values. The second step, called oblique analysis, aims to estimate factor curves representing the fundamental curves. In the case of multispectral analysis, factor curves are selected by positive constraints to obtain positive factor curves and images. Factor images are recomputed back to the original sampling by oblique projection on the factor curves, and the estimation is performed in the leastsquares sense. An algorithmic description of FAMIS is provided in a previous publication. ${ }^{16}$ This presentation is an adaptation of a published figure reprinted from International Journal of Nanomedicine, 5, Kahn E, Baarine M, Pelloux S, et al. Iron nanoparticles increase 7-ketocholesterol-induced cell death, inflammation, and oxidation on murine cardiac HLI-NB cells, I85-195, copyright (2010), with permission from Dove Medical Press Ltd. ${ }^{10} \mathrm{It}$ mentions excitation profiles, and images and factor curves are different. Abbreviation: FAMIS, factor analysis of medical image sequences. 
images were recomputed back to the original sampling by oblique projection on the factor curves. The estimation was performed in the least-squares sense; in this case, the factor curves correspond to the emission or excitation spectra of the fluorochromes. Factor images provide images of stained fluorescent structures. Two or more factor curves and factor images can be investigated. Superimposition of these factor images in their true colors provides a supplementary tool for result interpretation.

\section{Results}

\section{Two-photon CLSM analysis of Texas Red-stained specimens FTR beads}

In the case of spectral observations of FTR beads in which emissions were collected through band-pass filters and then processed before interpretation, excitation was performed at $805 \mathrm{~nm}$ and emission was collected in the spectral mode in $10 \mathrm{~nm}$ filters from blue to red (398-718 nm). Here, two factors only were analyzed. A red emission $(608 \mathrm{~nm})$ corresponding to Texas Red was visualized in one factor image, and a green emission (498 nm) corresponding to autofluorescence and to FITC was visualized in the second factor image (Figure 2A). When excitations were collected in the spectral mode ranging from 705 to $965 \mathrm{~nm}$, a $795 \mathrm{~nm}$ excitation peak corresponding to Texas Red was visualized in one factor image and a 745-765 nm band of excitations, corresponding to FITC and autofluorescence, was visualized in another (Figure 2B).

\section{MACS Texas Red Fe nanoparticles}

MACS Texas Red Fe nanoparticles were excited at $805 \mathrm{~nm}$ and emissions were collected in the spectral mode through $10 \mathrm{~nm}$ band-pass filters from blue to red (398-718 nm) and processed before interpretation. Here, two factors only were analyzed. A red emission (608 nm) corresponding to Texas Red was visualized in a first factor image, and a blue emission (448 nm) corresponding to background autofluorescence was visualized in the second factor image (Figure 3A). Excitations were also collected in the spectral mode ranging from 705 to $965 \mathrm{~nm}$; an excitation ranging between 795 and $825 \mathrm{~nm}$ corresponding to Texas Red was visualized in one factor image and a 745-765 band of excitations corresponding to the background autofluorescence in another (Figure 3B).

\section{Injected MRC Texas Red Fe nanoparticles}

For the injected MRC Texas Red Fe nanoparticles, excitations were performed at both $785 \mathrm{~nm}$ and 745 or $755 \mathrm{~nm}$, and emissions were again collected in the spectral mode in
$10 \mathrm{~nm}$ filters from blue to red (398-718 nm). Excitations were collected in the spectral mode ranging from $705 \mathrm{~nm}$ to $965 \mathrm{~nm}$, and the resulting spectral sequences were also investigated. Sequences of images showing emissions and excitations, respectively, were acquired from a control sample (Figure 4A and B), from a SYTO13 counterstained sample from a noninjected mouse (Figure $4 \mathrm{C}$ and $\mathrm{D}$ ), from a sample that was not counterstained taken from an injected mouse (Figure 4E and F), and from a counterstained sample taken from an injected mouse (Figure 4G and $\mathrm{H}$ ).

Here, two or three factors were analyzed (three factors in the cases of Figure 4A, E, and G). A green emission (498-518 nm) corresponding to autofluorescence (Figure 4A) or SYTO13 (Figure 4C and G) was visualized in the first factor image of the spectral emission sequences, and a red emission (598-638 nm band of emission, $628 \mathrm{~nm}$ peak) corresponding to Texas Red was visualized in the second factor image (Figure 4E and G). A blue emission (448-468 nm) corresponding to autofluorescence was visualized in the third factor image (Figure 4A, E, and G).

Excitations of $745 \mathrm{~nm}$ and $805 \mathrm{~nm}$, corresponding to autofluorescence and SYTO13, were visualized in the first factor image in Figure 4B, D, and H, and an excitation of $785 \mathrm{~nm}$, corresponding to Texas Red, was visualized in the second factor image in Figure 4D, F, and H.

\section{Analysis of results}

Considering that excitation is recommended ${ }^{14}$ at $780 \mathrm{~nm}$ for Texas Red, between $740 \mathrm{~nm}$ and $820 \mathrm{~nm}$ for FITC, and at $810 \mathrm{~nm}$ for SYTO13, there is a good concordance of the results that were obtained for Texas Red in FTR beads, MACS Texas Red Fe nanoparticles, and injected MRC Texas Red Fe nanoparticles. The red emission was at $608 \mathrm{~nm}$ and the corresponding excitations were at $795 \mathrm{~nm}$, between 795 and $825 \mathrm{~nm}$, and at $785 \mathrm{~nm}$, respectively. SYTO13 (green emission at $498 \mathrm{~nm}$ ) was properly distinguished by its excitation in our results both from autofluorescence ( $805 \mathrm{~nm}$ ) and from FITC (excitation between 745 and $775 \mathrm{~nm}$ ). These results support the choice of Texas Red to stain structures and SYTO13 for counterstaining to determine the precise localization of structures in arterial wall histological tissue sections (thoracic aorta of mice) by using computed curves (factors) and corresponding factor images.

\section{Discussion}

The present study, performed on mice injected with Fe nanoparticles stained with Texas Red, was carried out to determine the ability of these nanoparticles to accumulate in the wall of the thoracic aorta. Preliminary studies 
A

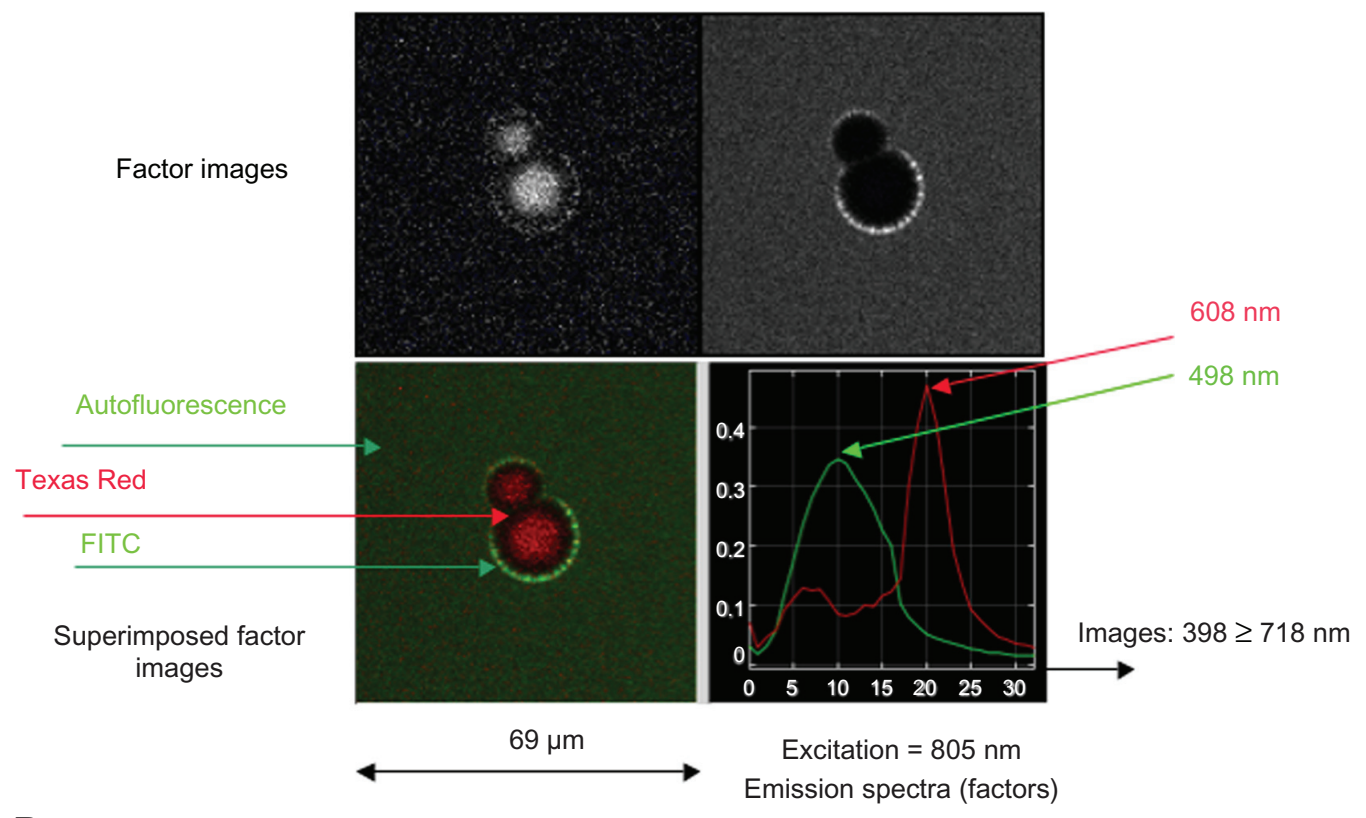

B

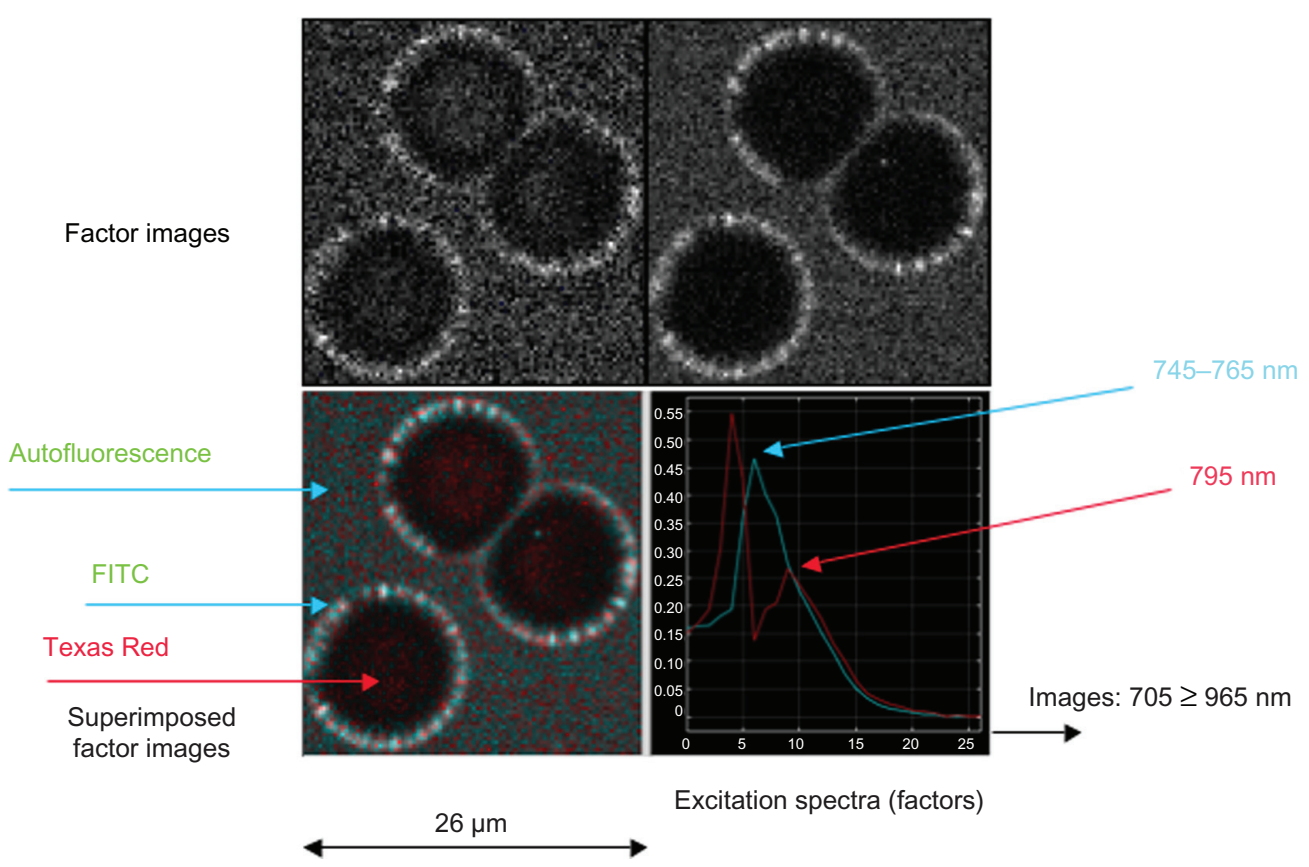

Figure 2 Spectral observations of FTR (FITC + Texas Red) beads in which (A) emission and (B) excitation sequences are collected and processed by FAMIS. Abbreviation: FAMIS, factor analysis of medical image sequences.

showed that Texas Red can be characterized by means of its excitation properties in calibrated conditions, ${ }^{11}$ and it was therefore possible to consider that Texas Red-stained nanoparticles could be identified in tissue sections on the basis of the fluorescent properties of this fluorochrome and autofluorescence. To this end, two-photon CLSM methods of investigation were used. As FITC is present in the FTR calibrated beads we used, we took the opportunity to show that FITC and Texas Red can be differentiated on the basis of their excitation properties; however, autofluorescence and FITC have similar factors and cannot be differentiated. Cell counterstaining with SYTO13 was also evaluated, and it resulted in proper differentiations. The fact that two-photon CLSMs are now equipped with tunable lasers enabled us to obtain excitation sequences of images, which was not possible in one-photon CLSMs. Confocal observations 
A

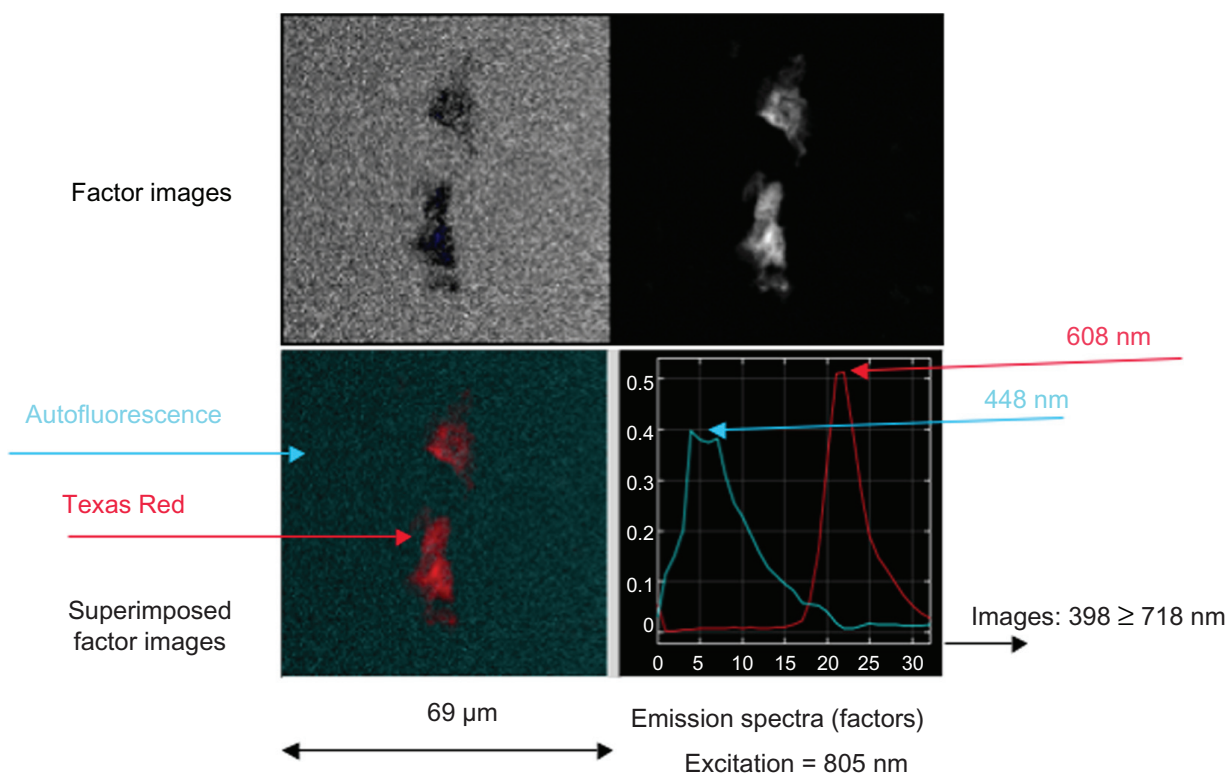

B

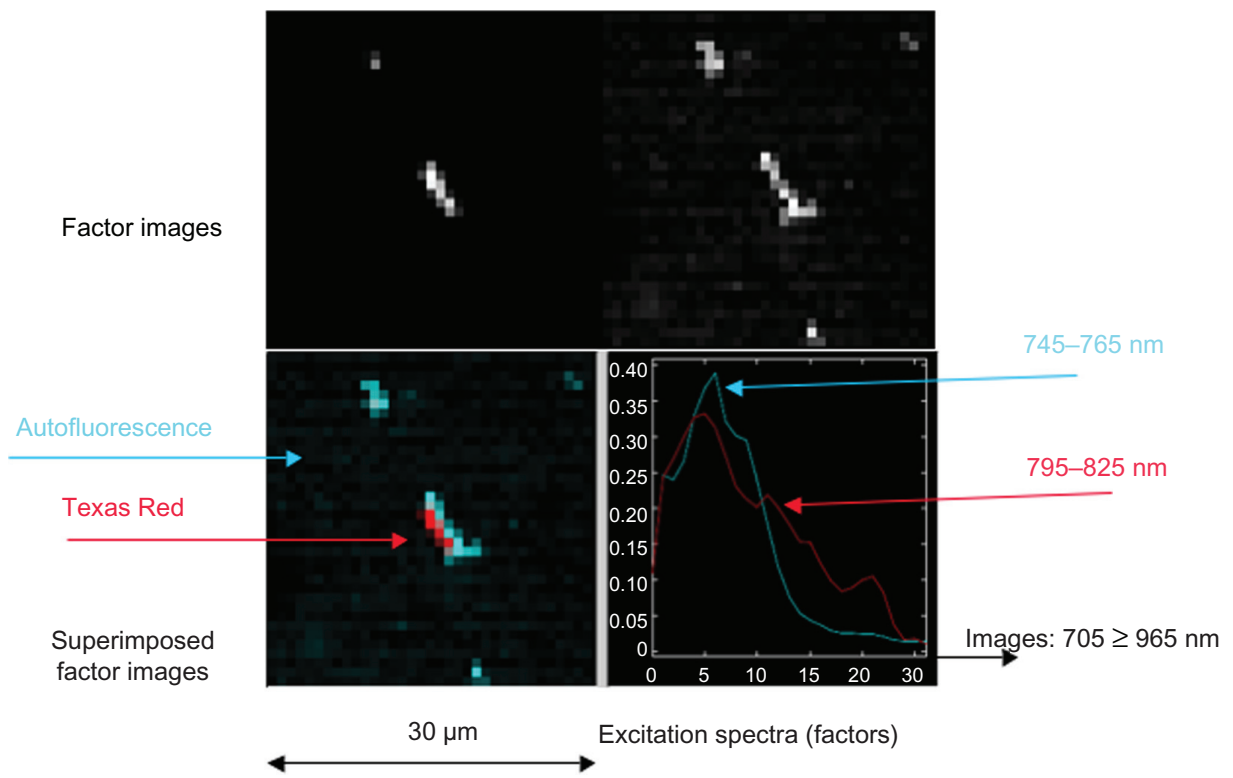

Figure 3 Spectral observations of iron nanoparticles conjugated with Texas Red (MACS Texas Red) in which (A) emission and (B) excitation sequences are collected through band-pass filters and processed by FAMIS.

Note: Color was added to assist with interpretation.

Abbreviation: FAMIS, factor analysis of medical image sequences.

were made more efficient and specific by using sequences of images provided by spectral excitation modes and then processed by image analysis. Indeed, using FAMIS as an unmixing tool makes it possible to differentiate spectral excitations or emissions of fluorochromes in a multistaining system and to provide images corresponding specifically to each fluorochrome. ${ }^{5}$ Compared to conventional microscopic methods of fluorescence - which require the use of specific sets of filters to deal with various technical constraints (fading, overlap of fluorescence, compensation) that often make it difficult to identify various objects inside specimens ${ }^{11}$ - the use of the FAMIS image-processing technique allows the differentiation of mixed fluorescent excitations or emissions from dyes and particles, ${ }^{19}$ while factor images provide an overview of the uptake of particles at the single-cell level. ${ }^{20}$ 
A

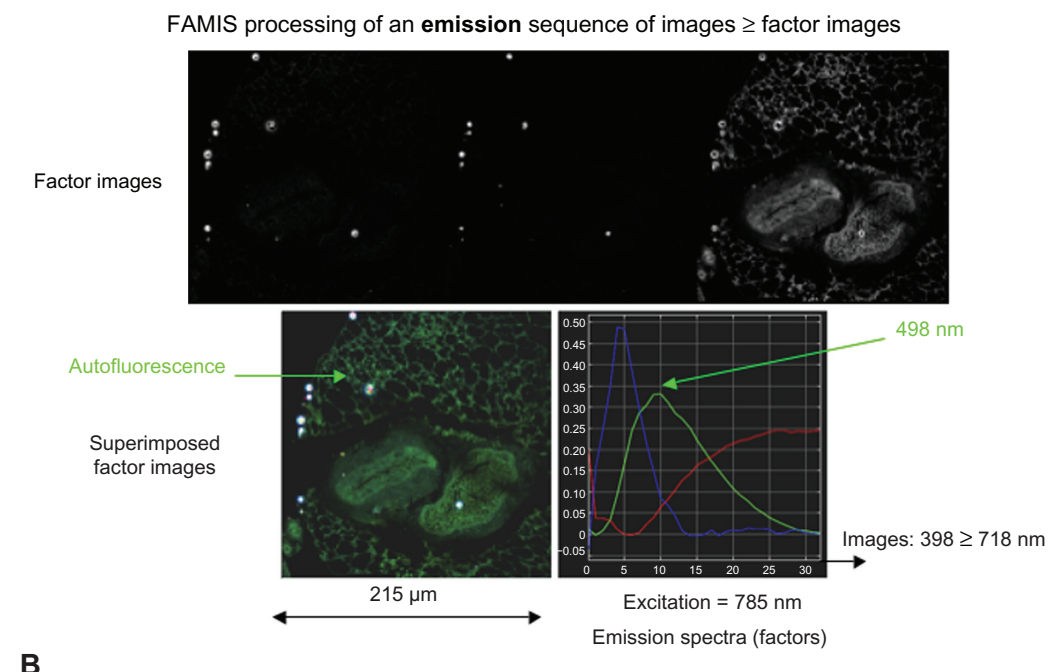

B

FAMIS processing of an excitation sequence of images $\geq$ factor images

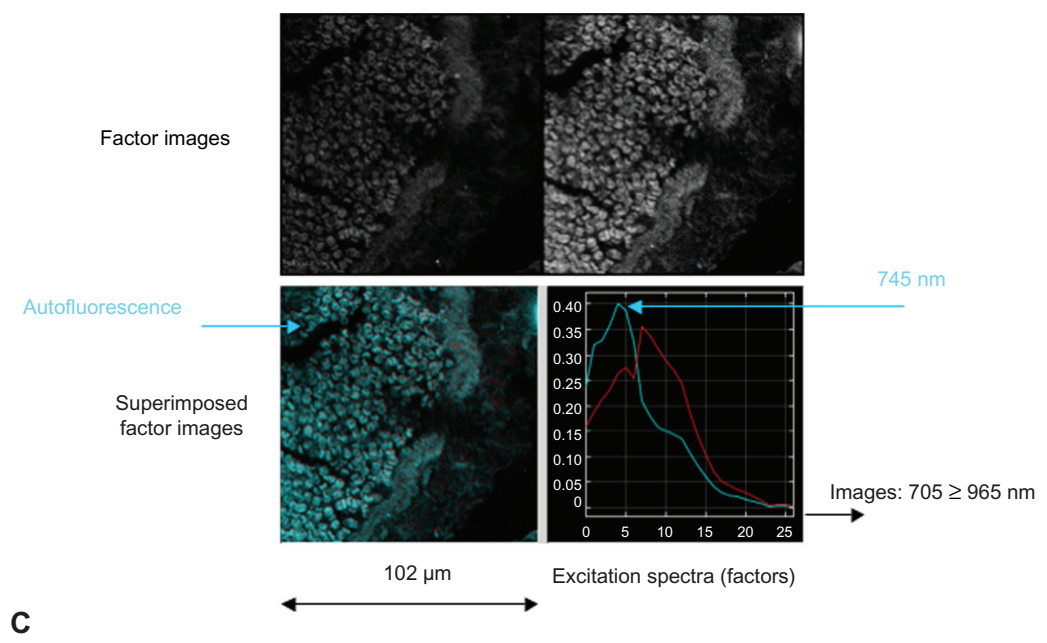

FAMIS processing of an emission sequence of images $\geq$ factor images

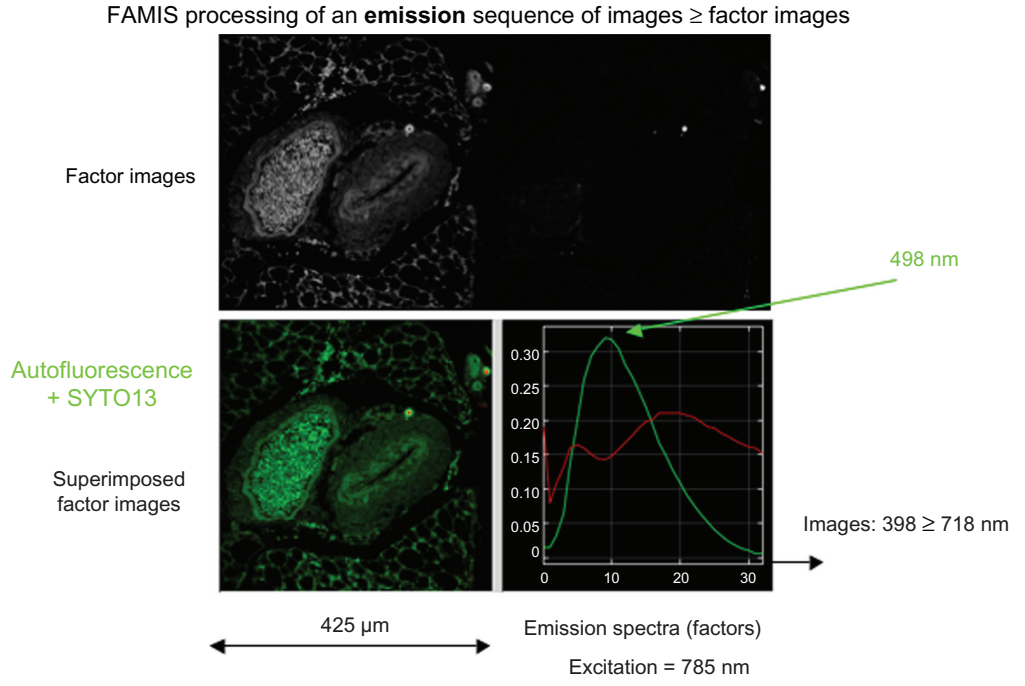

Figure 4 (Continued) 
D

FAMIS processing of an excitation sequence of images $\geq$ factor images

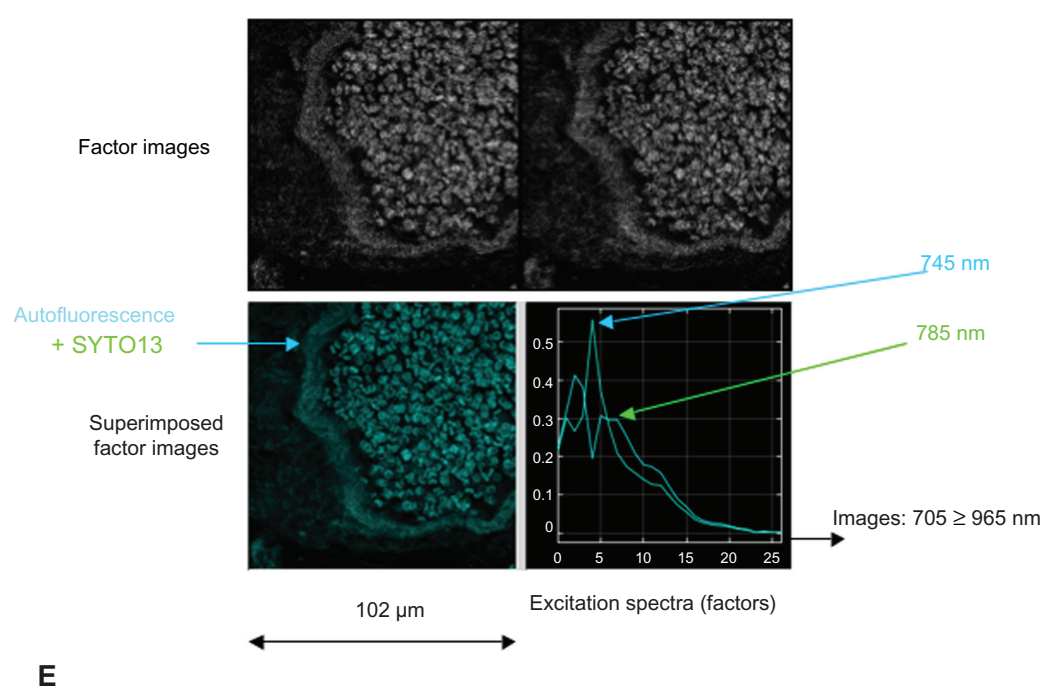

FAMIS processing of an emission sequence of images $\geq$ factor images

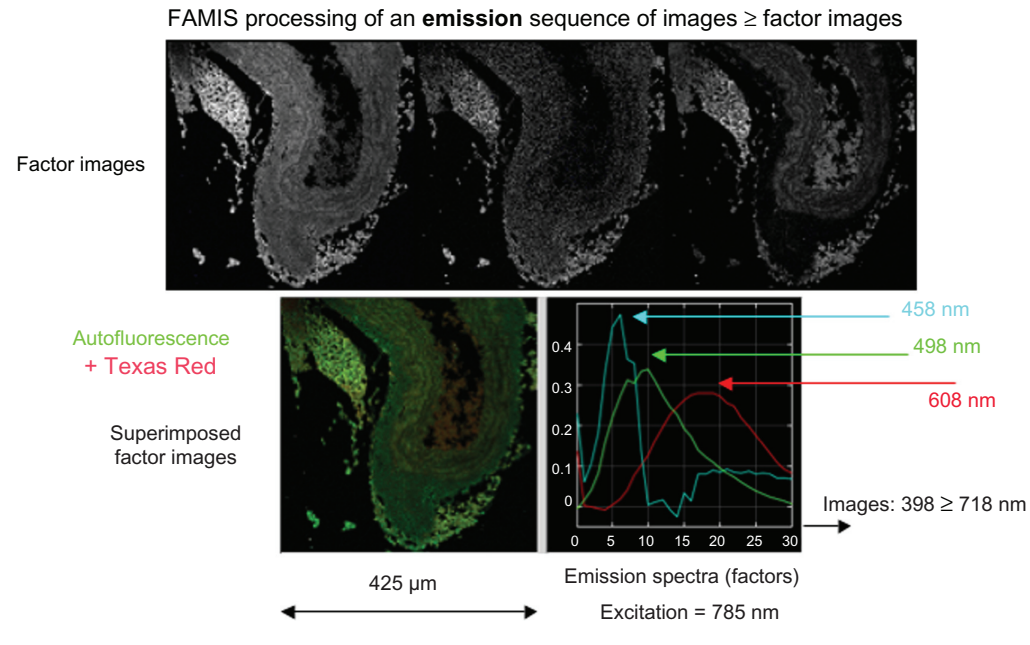

$\mathbf{F}$

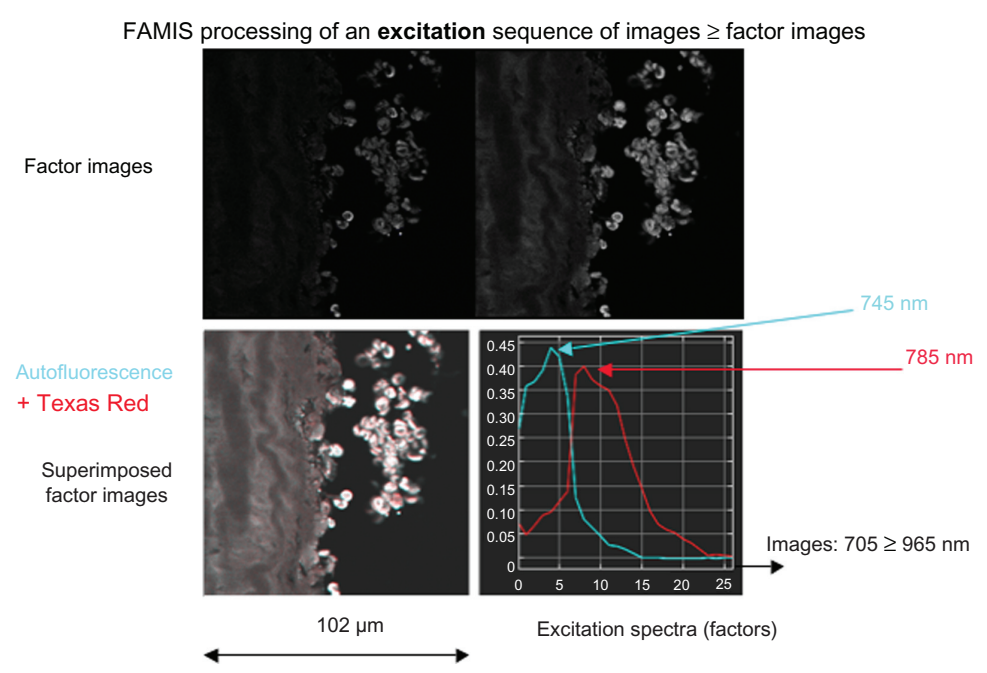

Figure 4 (Continued) 


\section{G}

FAMIS processing of an emission sequence of images $\geq$ factor images

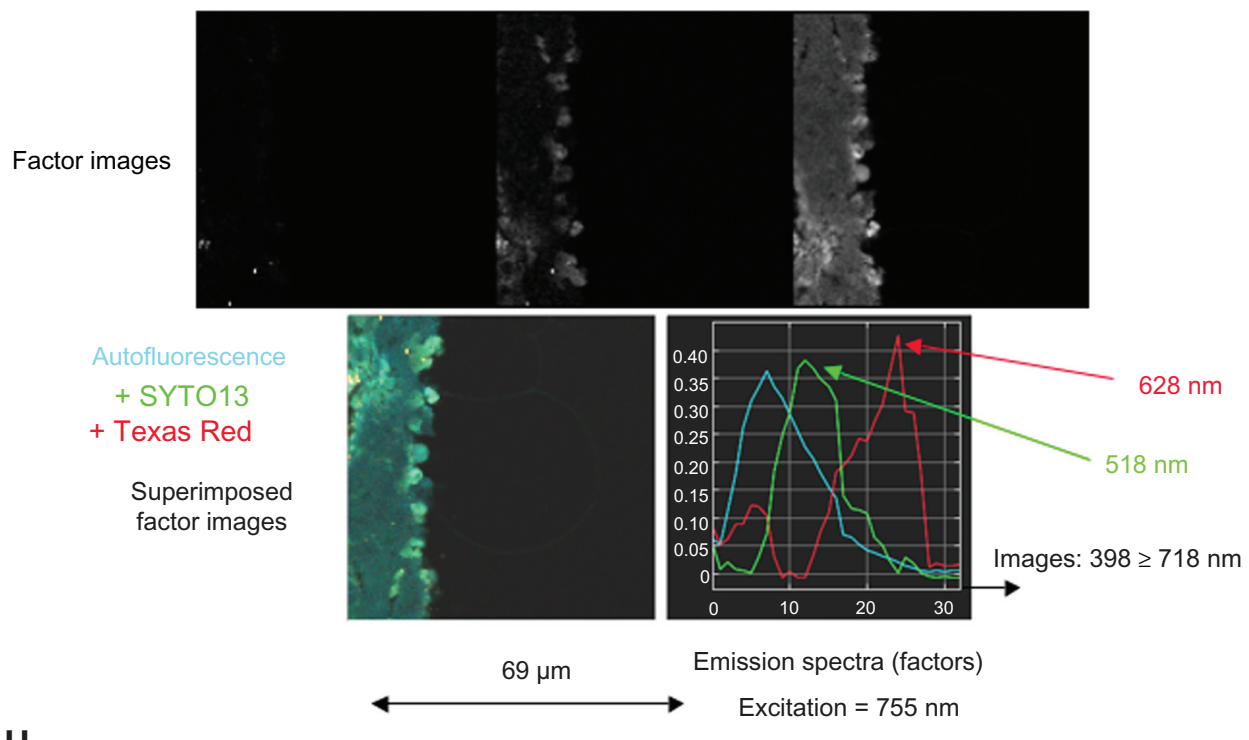

H

FAMIS processing of an excitation sequence of images $\geq$ factor images

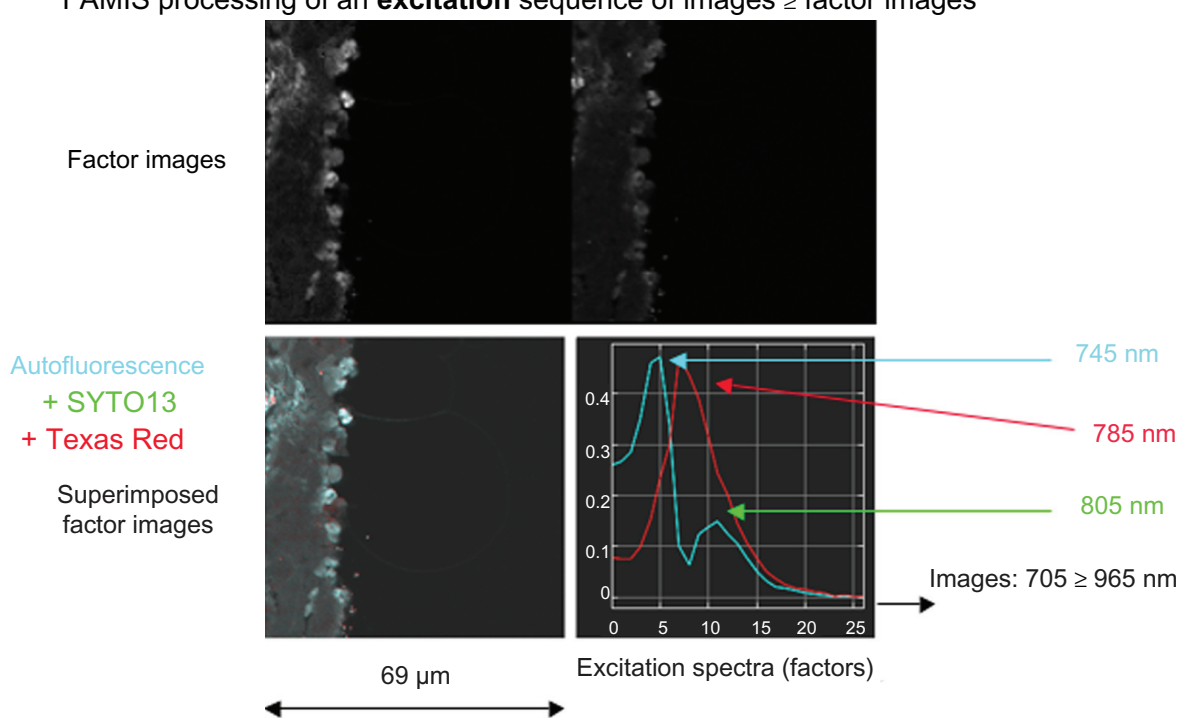

Figure 4 Spectral observation via FAMIS of the action of injected MRC Texas Red iron nanoparticles in the thoracic aorta of mice, including (A and $\mathbf{B})$ control samples with no injection and no staining; (C and $\mathbf{D}$ ) control samples counterstained with SYTOI3; (E and $\mathbf{F}$ ) injected samples with no staining; and ( $\mathbf{G}$ and $\mathbf{H})$ injected, counterstained samples. Notes: Of each pair, the former shows emissions (398-718 nm) and the latter shows excitations (705-965 nm). Color was added to assist with interpretation.

Abbreviation: FAMIS, factor analysis of medical image sequences.

In conclusion, the use of a two-photon CLSM equipped with a tunable laser, in conjunction with subsequent spectral methods of unmixing based on excitation properties of fluorochromes such as Texas Red and SYTO13, provides an efficient supplementary tool to analyze the interaction and the capture of stained nanoparticles by living cells or animals. Other fluorochromes can also be evaluated using this approach. Prospective works in vivo and ex vivo can therefore be considered to characterize side effects of various types of nanoparticles. ${ }^{21}$ The proposed approach combines spectral methods based on excitation with the use of a two-photon CLSM associated with appropriate biochemical and immunological methods. It opens the way to investigate fluorescent nanoparticles stained with 
different dyes and allows the investigation of the cellular and tissular distribution of various molecules associated with fluorescent nanoparticles and appropriate counterstainings. This approach could have some pharmacological and toxicological applications, resulting from obtaining the distribution of nanoparticles in various tissues in small animal models and in humans. ${ }^{22-24}$

\section{Acknowledgments}

This work was supported by the Institut National de la Santé et de la Recherche Médicale (INSERM); the Université de Bourgogne (Dijon, France); the Regional Council of Burgundy; and the Action Intégrée Franco-Marocaine CMIFM-AIMA/10/238 of the PAI Volubulis program, Ministère des Affaires Etrangères.

\section{Disclosure}

The authors declare no conflicts of interest.

\section{References}

1. Grosberg L, Radosevich A, Asfasha S, Wang T, Hillman E. Spectral characterization and unmixing of intrinsic contrast in intact normal and diseased gastric tissues using hyperspectral two-photon microscopy. PLoS One. 2011;6(5):e19925.

2. Di Paola R, Bazin J-P, Aubry F, et al. Handling of dynamic sequences in nuclear medicine. IEEE Trans Nucl Sci. 1982;29:1310-1321.

3. Frouin F, Cinotti L, Benali H, et al. Extraction of functional volumes from medical dynamic volumetric datasets. Comp Med Imaging Graph. 1993;17:397-404.

4. Vejux A, Lizard G, Tourneur Y, Riedinger JM, Frouin F, Kahn E. Effects of caspase inhibitors (z-VAD-fmk, z-VDVAD-fmk) on Nile Red fluorescence pattern in 7-ketocholesterol-treated cells: investigation by flow cytometry and spectral imaging microscopy. Cytometry A. 2007;71: $550-562$.

5. Kahn E, Hotmar J, Frouin F, et al. Spectral and dynamic confocal fluorescence characterization of cytogenetic preparations by factor analysis. Anal Cell Pathol. 1996;12:45-56.

6. Kahn E, Receveur A, Coullin P, et al. Multiple excitation confocal analysis of targets in nuclei of cytogenetic preparations. Anal Quant Cytol Histol. 2004;26:1-6.

7. Garini Y, Young IT, McNamara G. Spectral imaging: principles and applications. Cytometry A. 2006;69:735-747.

8. Radosevich A, Bouchard M, Burgess S, Chen B, Hillman E. Hyperspectral in vivo two-photon microscopy of intrinsic contrast. Opt Lett. 2008;33:2164-2166.
9. Kahn E, Lizard G, Dumas D, et al. Analysis of fluorescent MRI contrast agent behavior in the liver and thoracic aorta of mice. Anal Quant Cytol Histol. 2004;26:233-238.

10. Kahn E, Baarine B, Pelloux S, et al. Iron nanoparticles increase 7-ketocholesterol-induced cell death, inflammation, and oxidation on murine cardiac HL1-NB cells. Int J Nanomedicine. 2010;5:185-195.

11. Kahn E. Confocal microscopy: characterization of fluorescent tracers by image processing of optical sections. Pathol Biol. 2001;49: 194-198.

12. Kawata S, Sasaki K, Minami S. Component analysis of spatial and spectral patterns in multispectral images I Basis. JOpt Soc Am A. 1987;4: 2101-2106

13. Mathies RA, Stryer L. Part 1-6: Single-Molecule Fluorescence Detection: a feasibility study using phycoerythrin. In: Lansing Taylor D, Waggoner AS, Murphy RF, Lanni F, Birge RR, editors. Applications of Fluorescence in the Biomedical Sciences. New York, NY: Liss; 1986: 129-140.

14. Diaspro A, Chirico G, Collini M. Two-photon fluorescence excitation and related techniques in biological microscopy. Q Rev Biophys. 2005; 38(2):97-166.

15. Kahn E, Frouin F, Souchier C, et al. Confocal multilaser focusing and single-laser characterization of UV excitable stains of cellular preparations. Cytometry A. 2000;40:42-49.

16. Kahn E, Lizard G, Pélégrini M, et al. Four-dimensional factor analysis of confocal images sequences (4D-FAMIS) to detect and characterize low numbers of human papillomavirus DNA by FISH in HeLa and SiHa cells. J Microscopy. 1999;193:227-243.

17. Harman HH. Modern Factor Analysis. Chicago, IL: University of Chicago Press; 1960.

18. Benzecri J-P. L'Analyse des Données, Tome 2: L'Analyse des Correspondances [Data Analysis, Vol 2: Correspondence Analysis]. Paris, France: Dunod; 1973. French.

19. Kahn E, Receveur A, Coullin P, et al. Multiple excitation confocal analysis of targets in nuclei of cytogenetic preparations. Anal Quant Cytol Histol. 2004;26:1-7.

20. Kahn E, Vejux A, Menetrier F, et al. Analysis of CD36 expression on human monocytic cells and atherosclerotic tissue sections with quantum dots. Investigation by flow cytometry and spectral imaging microscopy. Anal Quant Cytol Histol. 2006;28:14-26.

21. Kayser K, Radziszowski D, Bzdyl P, Sommer R, Kayser G. Towards an automated virtual slide screening: theoretical considerations and practical experiences of automated tissue-based virtual diagnosis to be implemented in the Internet. Diagn Pathol. 2006;10:1-10.

22. Roberts MS, Dancik Y, Prow TW, et al. Non-invasive imaging of skin physiology and percutaneous penetration using fluorescence spectral and lifetime imaging with multiphoton and confocal microscopy. Eur J Pharm Biopharm. 2011;77(3):469-488.

23. Pitsillides CM, Runnels JM, Spencer JA, Zhi L, Wu MX, Lin CP. Cell labeling approaches for fluorescence-based in vivo flow cytometry. Cytometry A. 2011;79:758-765.

24. Song Y, Tang S. Nanoexposure, unusual diseases, and new health and safety concerns. Scientific World Journal. 2011;11:1821-1828.
International Journal of Nanomedicine

\section{Publish your work in this journal}

The International Journal of Nanomedicine is an international, peerreviewed journal focusing on the application of nanotechnology in diagnostics, therapeutics, and drug delivery systems throughout the biomedical field. This journal is indexed on PubMed Central, MedLine, CAS, SciSearch $\AA$, Current Contents ${ }^{\circledR} /$ Clinical Medicine,

\section{Dovepress}

Journal Citation Reports/Science Edition, EMBase, Scopus and the Elsevier Bibliographic databases. The manuscript management system is completely online and includes a very quick and fair peer-review system, which is all easy to use. Visit http://www.dovepress.com/ testimonials.php to read real quotes from published authors. 